\title{
Separating visible persistence from retinal afterimages
}

\author{
VINCENT Di LOLLO \\ University of Alberta, Edmonton, Alberta, Canada \\ and \\ CRAIG D. CLARK and JOHN H. HOGBEN \\ University of Western Australia, Nedlands, Western Australia, Australia
}

\begin{abstract}
Visible persistence (the continued visibility of a brief display for a few hundred milliseconds after stimulus termination) has sometimes been described as a weak, short-lived retinal afterimage. An opposite viewpoint holds that visible persistence and retinal afterimages are separate phenomena. In a series of three experiments, we employed a temporal integration task to compare visible persistence and retinal afterimages directly under identical viewing conditions. The task required perceptual integration of two successive displays across a brief temporal gap. It was found that visible persistence and retinal afterimages have different rates of decay across a temporal gap (Experiment 1), and that the two are affected differently by changes in duration of the leading display (Experiment 2) and by changes in the intensity of both displays (Experiment 3). We conclude that visible persistence and retinal afterimages are separate phenomena, supported by different mechanisms within the visual system.
\end{abstract}

A brief visual display may remain visible for $100 \mathrm{msec}$ or longer after termination of the physical stimulus. The additional period of visibility is known as visible persistence.

Although this phenomenon has been extensively investigated (see reviews by Coltheart, 1980; Long, 1980), there is no broad agreement as to its precise nature. While some investigators regard it as akin to a weak, short-lived retinal afterimage (e.g., Long \& Sakitt, 1980; Sakitt, 1976), others consider it to be a separate perceptual effect that has quite distinct properties (Breitmeyer, 1984; Coltheart, 1980; Di Lollo, 1980; Eriksen \& Collins, 1967). Although there is little doubt that afterimages are of retinal origin (Brindley, 1962; Craik, 1940), a more central locus has been suggested for visible persistence (Banks \& Barber, 1977; Meyer \& Maguire, 1977). Evidence from separate experiments suggests that, indeed, the two may respond differently to manipulation of specific stimulus variables and may therefore be regarded as distinct phenomena. For example, the duration of retinal afterimages has been reported to be positively related to both the intensity and energy of the inducing stimulus (Brown, 1965; Uttal, 1981). By contrast, there is evidence to suggest that the duration of visible persistence is inversely related to these stimulus variables (Coltheart,

This research was supported by Grant A6592 from the Natural Sciences and Engineering Research Council of Canada to Vincent Di Lollo, and by Grant 8515045 from the Australian Research Grants Scheme to John Hogben. We thank Charles Bourassa, Peter Dixon, and Robert Efron for commenting on an earlier version of this paper. Requests for reprints should be addressed to Vincent Di Lollo, Department of Psychology, University of Alberta, Edmonton, Alberta T6G 2E9, Canada.
1980), although contrary evidence has also been reported (Long \& Sakitt, 1980; Sakitt, 1976).

While clearly suggestive, this evidence cannot be regarded as definitive, because the two phenomena have invariably been studied in isolation under experimental conditions that differed in respect to such fundamental variables as degree of dark adaptation and range of stimulus values. For direct comparison, the two must be studied under the same experimental conditions over a common range of stimulus values.

A single paradigm and a common range of stimulus values were employed in the present work to compare the temporal courses of visible persistence and retinal afterimages. Duration of these aftereffects was studied with the method of temporal integration of form. Frequently used for this purpose (e.g., Eriksen \& Collins, 1967; Hogben \& Di Lollo, 1974), this method requires perceptual integration of two brief sequential displays, separated by a temporal gap. In the present work, the basic display consisted of a $5 \times 5$ square matrix of light elements flashed on an oscilloscope. One of the elements, chosen randomly on each trial, was not shown. The observer's task was to name the matrix coordinates of the missing element.

To study visible persistence, the 24 matrix elements are displayed in two successive frames of 12 elements each, separated by a temporal gap (interstimulus interval, ISI). At brief ISIs, the two frames can be easily integrated, and the location of the missing element readily identified. However, at longer ISIs, temporal integration fails and the two frames are seen as separate configurations. In this task, the missing element cannot be located unless the two portions of the display are seen as one. In turn, perceptual overlap requires some form of continuing visibility 
of the leading stimulus capable of bridging the ISI. The longest ISI at which the two frames can still be integrated provides an index of the duration of visible persistence.

In the course of investigating temporal integration under conditions of dark adaptation, we noted a curious phenomenon apparent to dark-adapted observers. At long ISIs, the two frames were seen as temporally discrete. Nevertheless, an integrated matrix could be seen-and the location of the missing element could be clearly identified-within an afterimage that became visible $1 \mathrm{sec}$ or so after the initial display. Thus, dark-adapted observers could perform the task either on the basis of the short-lived visible persistence produced by the leading display and/or on the basis of the more durable afterimage that became visible about $1 \mathrm{sec}$ later.

It occurred to us that this observation offered a means of investigating visible persistence and retinal afterimages separately, yet under identical experimental conditions. This was done by requiring observers to perform the matrix-integration task on the basis of either the shortlived visible persistence or the later afterimage. In a series of three experiments, we studied how the time courses of visible persistence and of retinal afterimages are affected by the duration of the temporal gap and by the duration and intensity of the inducing stimuli.

\section{EXPERIMENT 1}

In Experiment 1, our aim was to compare temporal integration of the two portions of the matrix across a range of ISIs, with and without the aid of retinal afterimages.

Preliminary trials had indicated that afterimages could support temporal integration across far longer ISIs than could visible persistence; therefore, we needed to remove the afterimage as a useful source of information whenever the response was to be made solely on the basis of visible persistence. In Experiment 1, this was achieved through a masking procedure: On the appropriate trials, all 25 matrix elements were flashed briefly immediately before the afterimage became visible, that is, about $1 \mathrm{sec}$ after the initial display. This procedure permitted the afterimage to be masked without affecting the initial display.

Two viewing conditions were employed. In one, the afterimage remained available as a basis for making the response; in the other, the afterimage was masked, and the response could be made only on the basis of visible persistence.

\section{Method}

Observers. A male observer naive to the purpose of the research and one of the authors served in all experiments. Both had normal or corrected-to-normal vision.

Displays. The X, Y, and Z (intensity) coordinates of all points to be displayed were stored in a fast plotting buffer capable of transferring them to an oscilloscope at the rate of $1,000 \mathrm{dots} / \mathrm{msec}$ (Finley, 1985). All stimuli were displayed on a Hewlett-Packard 1333A oscilloscopic point plotter equipped with P15 phosphor. At the view- ing distance of $57 \mathrm{~cm}$, one side of the $8 \times 8 \mathrm{~cm}$ display surface subtended a visual angle of $8^{\circ}$.

The display consisted of 24 of the 25 elements defining a $5 \times 5$ square matrix. Each element consisted of a filled light square that subtended $0.1^{\circ}$ of visual angle, and was separated edge-to-edge from adjacent elements by $0.2^{\circ}$. The matrix as a whole formed a square with sides of $1.3^{\circ}$. The stimuli were displayed at a luminance of $8.9 \mathrm{~cd} / \mathrm{m}^{2}$, corresponding to a retinal illuminance of approximately 500 Td.

Procedure. At the beginning of each session, the observer was dark-adapted for at least $10 \mathrm{~min}$ in a lightproof viewing chamber. All displays were viewed binocularly with natural pupils, with the viewing distance set by a headrest. At the beginning of each trial, four dim fixation points were displayed at the corners of a $2^{\circ}$ square area in the center of the display surface. The observer pressed a button to initiate a trial consisting of the following sequence of events: first, 12 matrix elements, chosen randomly on each trial, were displayed for $10 \mathrm{msec}$; next, an ISI elapsed, lasting 10, 20, 40,80 , or $160 \mathrm{msec}$; finally, another 12 elements (chosen randomly from the remaining 13 matrix locations) were displayed for $10 \mathrm{msec}$, and the fixation points were turned off. The observer then identified the location of the missing element, guessing if unsure, and encoded its row and column coordinates by means of a button box connected to a computer that performed all timing and scoring functions. The fixation points then reappeared to indicate readiness for the next trial. A minimum interval of $5 \mathrm{sec}$ was enforced between successive trials.

There were two types of trials, "masking" and "no-masking." On no-masking trials, the sequence of events was as described above, with the observer being free to base his response on either visible persistence or the retinal afterimage. On masking trials, a 10-msec display of all 25 matrix elements was presented $800 \mathrm{msec}$ after the termination of the second frame. This procedure masked the oncoming afterimage and forced the observer to base his response on visible persistence alone.

On some no-masking trials, no afterimage was obtained, and the observer was permitted to abort the trial by pressing a button. Similarly, on some masking trials, the observer made an unintentional eye movement that averted masking of the afterimage. Again, in this case, the observer could abort the trial. The total number of aborted trials was less than $10 \%$ for each observer.

An experimental run consisted of 100 trials in either the masking or the no-masking condition. The 100 trials were ordered randomly for each run and comprised 20 presentations of the dot matrix at each of the five ISIs. Each observer served in 10 experimental runs, 5 each in the masking and the no-masking conditions. One experimental session comprised three runs, and lasted about $1 \mathrm{~h}$.

\section{Results and Discussion}

The results are illustrated in Figure 1, separately for each observer. Each point in Figure 1 is based on 100 observations.

Clearly, when the observers could utilize the afterimages (no-mask condition), performance was virtually errorless, and remained so at all ISIs. By contrast, when the afterimage was unavailable (mask condition), performance was errorless at the short ISIs but deteriorated rapidly as ISI was lengthened. This strongly suggests that at least two factors were at work to mediate temporal integration of the matrix. The first, retinal afterimages, could support integration throughout the range of ISIs; however, when afterimages were removed 


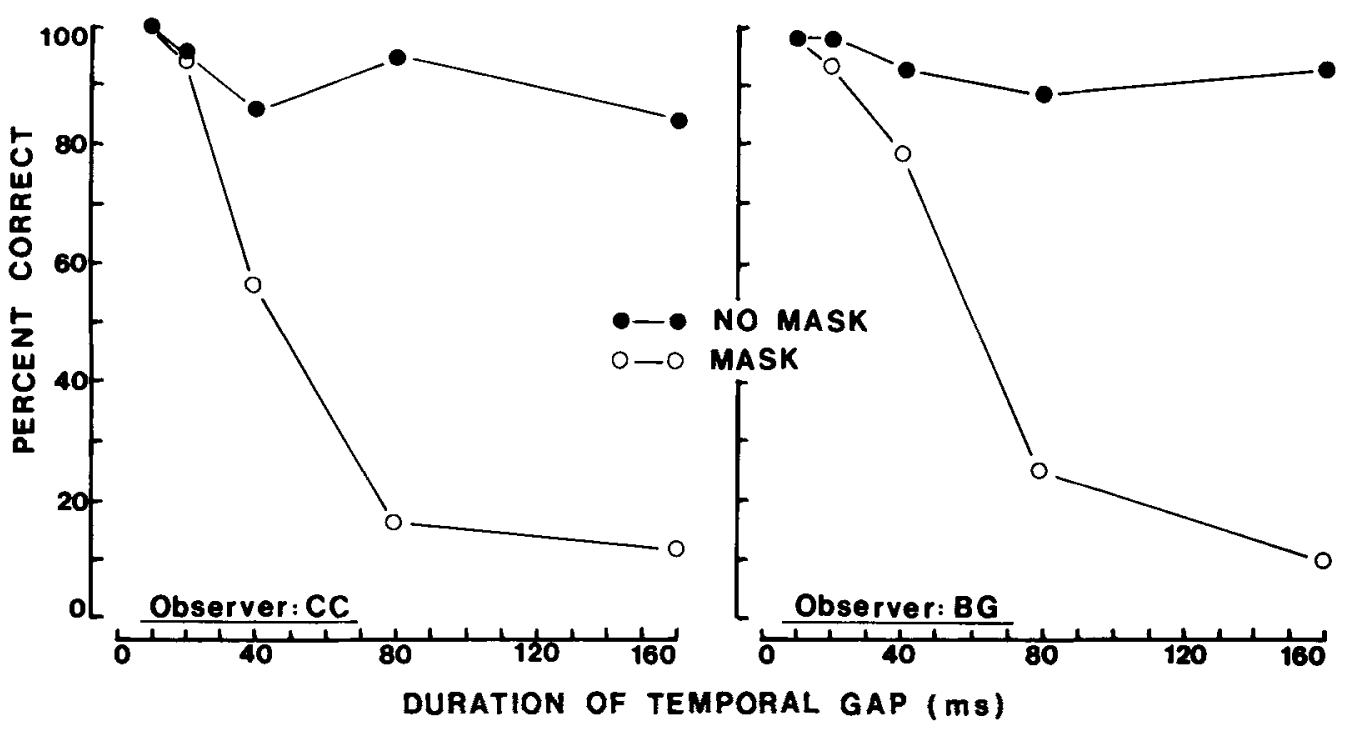

\begin{abstract}
Figure 1. Percentage of correct responses in identifying the location of a missing element within a $5 \times 5$ element square matrix displayed in two portions of 12 elements each, separated by an ISI whose duration varied as shown on the abscissa. In the condition labeled "no mask," the dark-adapted observer identified the location of the missing element from the afterimage that became visible about 1 sec later. In the condition labeled "mask," the afterimage was masked by a 10-msec display of all 25 matrix elements 800 msec after the initial display.
\end{abstract}

as a source of information, another factor was available to mediate temporal integration at the shorter, but not at the longer, ISIs. In agreement with current usage, we refer to this process as visible persistence, whose time course has been shown in numerous related studies to be indistinguishable from the lower curves seen in Figure 1. While the present range of stimulus onset asynchronies encompassed the time course of visible persistence, it was too narrow to span the duration of retinal afterimages. Obviously, the no-mask curves in Figure 1 would require far longer ISIs to reach asymptote. Longer ISIs, however, are not suited to the present paradigm. As ISI increases, so does the likelihood that an eye movement will occur during the display sequence and disrupt perceptual integration. However, not tracking the temporal decay of the afterimages did not affect the major purpose of this study, which was to distinguish visible persistence from retinal afterimages as a basis for temporal integration. This distinction is clearly illustrated in Figure 1.

It must be stressed that, because the 800 -msec delay between display and mask far exceeded the range of delays over which backward masking remains effective (Breitmeyer, 1984; Uttal, 1981), the masking procedure employed in this study permitted the afterimage to be removed as a source of information without affecting perception of the initial display. That perception of the initial display was not influenced by the mask is evidenced by the high level of performance seen in Figure 1 for the mask condition at brief ISIs.

Having found that visible persistence and retinal afterimages are affected in different ways by a gap in the display sequence, we now examine a second variable known to affect temporal integration, namely, the duration of the leading display.

\section{EXPERIMENT 2}

Duration of visible persistence decreases as the exposure duration of the inducing stimulus is increased. Known as the inverse duration effect, this relationship has been obtained under a wide range of conditions (Coltheart, 1980). By contrast, duration of positive afterimages is known to vary with the energy rather than with the duration of brief stimuli (e.g., Brown, 1965). Although reliably obtained, these effects invariably have been studied in unrelated experiments under widely different viewing conditions.

The purpose of Experiment 2 was to examine how changes in exposure duration of the inducing stimulus affect the durations of visible persistence and retinal afterimages under the same viewing conditions.

\section{Method}

The method and procedures in Experiment 2 were essentially the same as those used in Experiment 1, except that the durations of the temporal gap and the trailing display were fixed at 20 and $40 \mathrm{msec}$, respectively. The duration of the leading display was 10 , $20,40,80$, or 160 msec.

Because of the time-intensity reciprocity known as Bloch's law (Uttal, 1981), brief displays that differ in duration are also normally seen to differ in brightness. In Experiment 2, brightness differences were eliminated as a confounding factor through a simple brightness-equalization procedure (Di Lollo \& Finley, 1986) by which shorter stimuli are presented at higher intensity levels, so that all displays appear of uniform brightness. In all other respects, the procedures were the same as in Experiment 1. 


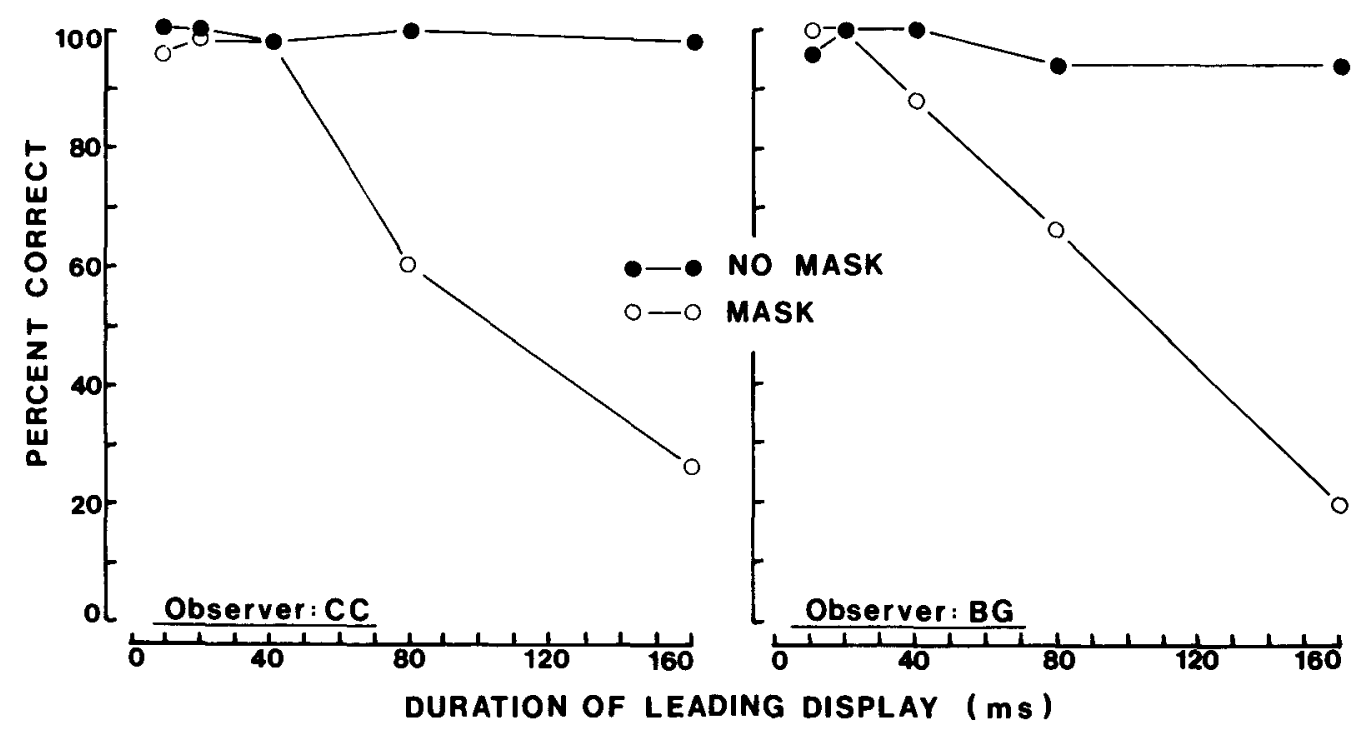

Figure 2. Percentage of correct responses in Experiment 2. The ISI was fixed at 20 msec, the trailing portion of the matrix was displayed for $\mathbf{4 0}$ msec, and the duration of the leading portion was varied as shown on the abscissa.

\section{Results and Discussion}

The results are illustrated in Figure 2, separately for each observer.

For both observers, performance based on visible persistence alone (mask condition) became progressively impaired as leading-stimulus duration was increased beyond about $\mathbf{4 0} \mathrm{msec}$. This is in keeping with the findings of earlier studies that employed similar paradigms and were conducted under conditions in which afterimages were not available (Allport, 1968; Di Lollo, 1980; Efron \& Lee, 1971). On the other hand, performance based on afterimages (no-mask condition) was not affected by increments in stimulus duration.

This pattern of results strongly suggests that perceptual integration in the two conditions was accomplished by processes that respond differently to stimulus duration. On this premise, visible persistence and retinal afterimages must be regarded as being based on separate mechanisms.

Declining performance in the mask condition is inconsistent with the view that visible persistence can be identified with the decaying contents of a sensory store (e.g., Neisser, 1967). A storage hypothesis assumes an image whose strength does not begin to decay until the end of stimulation. Given a temporal gap of constant duration, a storage hypothesis cannot explain how the gap could be bridged by an image produced by a brief $10-\mathrm{msec}$ stimulus but not by a stimulus 16 times longer. An alternative hypothesis, based on an active process of fixed duration, time-locked to the onset of stimulation, has been described elsewhere (Di Lollo, 1980) and is considered below.

\section{EXPERIMENT 3}

The outcomes of Experiments 1 and 2 indicate that visible persistence and retinal afterimages are affected differ- ently by changes in ISI and in exposure duration. There is evidence to suggest that the two phenomena may be separable on the basis of yet another variable, namely, stimulus intensity.

Evidence from separate and unrelated studies suggests that increments in intensity prolong retinal afterimages (Brown, 1965) but reduce the duration of visible persistence (Allport, 1968).

Experiment 3 was designed to examine the role of stimulus intensity on the durations of visible persistence and retinal afterimages under common viewing conditions.

\section{Method}

The method and procedures were essentially the same as in Experiment 1, except that in Experiment 3 the ISI was fixed at $60 \mathrm{msec}$ and stimuli were displayed at one of two intensities, $0.2 \mathrm{~cd} / \mathrm{m}^{2}$ (approximately $11 \mathrm{Td}$ ) or $8.9 \mathrm{~cd} / \mathrm{m}^{2}$ (approximately $500 \mathrm{Td}$ ). The two halves of each display, as well as the mask, were always of equal intensity, and their duration was always $10 \mathrm{msec}$.

While the bright stimuli produced afterimages as in Experiments 1 and 2 , the dim stimuli failed to produce any visible afterimages. Accordingly, data were not collected for the no-mask condition with dim stimuli. The experiment thus comprised three conditions: a nomask condition with bright stimuli, which allowed observers to employ afterimages, and two mask conditions (one with bright, the other with dim stimuli) in which the task could be performed only on the basis of visible persistence.

\section{Results and Discussion}

The outcome of Experiment 3 is shown in Figure 3, which contains the results for the three experimental conditions.

These results distinguish visible persistence from retinal afterimages in two ways. Considering first the results with the dim stimuli, it is clear that temporal integration based on visible persistence was almost perfect with stimuli that were too dim to produce visible afterimages, that is, with stimuli whose energy, while certainly suffi- 


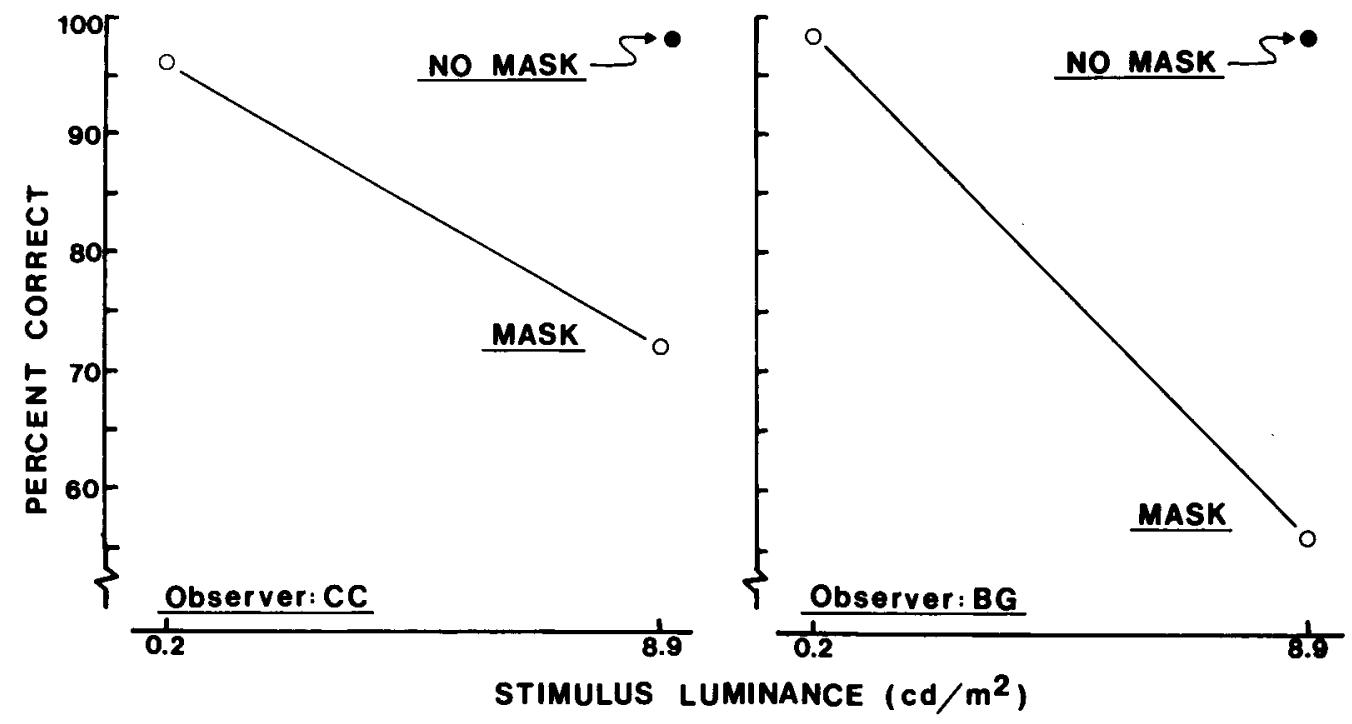

Figure 3. Percentage of correct responses in Experiment 3. The two portions of the matrix were shown for 10 msec each at the same intensity of either 0.2 or $8.9 \mathrm{~cd} / \mathrm{m}^{2}$. The ISI was constant at 60 msec.

cient for initiating the activity underlying visible persistence, was obviously insufficient for saturating the photoreceptors to the point of producing a viable afterimage. This strongly suggests that persistence and afterimages are based on separate mechanisms that respond differently to stimulus intensity.

The results with the bright stimuli (Figure 3) show that while an increment in intensity was beneficial to the development of afterimages, it was detrimental to visible persistence, as has been found in other investigations (Adelson \& Jonides, 1980; Coltheart, 1980; Irwin \& Yeomans, 1986). Again, this is strongly suggestive of separate mechanisms.

\section{GENERAL DISCUSSION}

Collectively, the evidence from all three experiments indicates that visible persistence and retinal afterimages are separate phenomena, supported by different mechanisms.

At least three differences between visible persistence and retinal afterimages were noted by the observers. First, the afterimages seen under the present display conditions were achromatic, whereas the integrated images based on visible persistence exhibited the blue-green appearance of the oscilloscope's phosphor. Second, there were substantial differences in latency: afterimages required about $1 \mathrm{sec}$ to emerge, but visible persistence had no discernible latency and was effectively a continuation of the physical display. Finally, there were marked differences in the ways in which the images disappeared: persistence ended abruptly, whereas afterimages remained visible for at least $1 \mathrm{sec}$ and were seen to fade progressively into the dark background.

The longer latency of the afterimages can be explained in terms of optical and retinal events: It is reasonable to surmise that the light energy from the stimulus bleaches the photoreceptors that correspond to the matrix elements and, through dispersion within the eye, induces a lesser degree of bleaching in the photoreceptors that correspond to the spaces between the elements. Initially, a fuzzy afterimage is produced by the continued activity of all stimulated receptors. Then, as the less-bleached receptors recover, the individual matrix elements become discernible. The observers' reports of the appearances of the displays are in complete agreement with this account.

On the face of it, an afterimage seems to have the salient attributes of a store whose contents grow with the energy of the inducing stimulus, and begin to decay when stimulation ends. This is consistent with Craik's (1940) classic experiment showing that high-intensity stimuli produce afterimages that are of retinal origin. More specifically, it is consistent with a process based on photoreceptor functioning.

By contrast, visible persistence cannot plausibly be regarded as being based on a similar store. Variations in stimulus duration and intensity affect degree of temporal integration in ways that are opposite to what might be expected from photoreceptor functioning. It has been suggested (Di Lollo, 1980) that visible persistence may be regarded as the outcome of sensory coding activity initiated at the onset of the stimulus. The activity is held to endure for a fixed period, resulting in visible persistence whose duration is time-locked to the onset of stimulation. This interpretation effectively decouples duration of visible persistence from that of the inducing stimulus, thus making perceptual integration dependent not on the temporal gap between two displays, but on the asynchrony between their onsets.

Whatever the underlying mechanisms, the inference to be drawn from the empirical evidence is unambiguous: Visible persistence and retinal afterimages cannot be regarded as belouging to the same class of events. Unquestionably, temporal integration can be achieved on the 
basis of either visible persistence or retinal afterimages; but the finding that the two respond quite differently to manipulations of the same variables under identical experimental conditions establishes them as clearly distinct phenomena.

\section{The Multiplicity of Visual Aftereffects}

At a most general level, the outcome of the present work disconfirms earlier claims that the "icon" (i.e., the continued visibility of a stimulus beyond its termination) is a unitary phenomenon (e.g., Sakitt, 1975). There would be little advantage in regarding the results of the mask and no-mask conditions as different expressions of the same category of events, even if the category is given a broad designation such as "afterimages." If one defines an afterimage as any residual information available after the stimulus is removed, then the early residual, which we have referred to as "visible persistence," is, by this definition, an afterimage. However, the point of the present work was to demonstrate that the characteristics of this early poststimulus persistence are quite different from those of the late poststimulus persistence (the classical afterimage). The present results indicate that the two types of persistence are not due to the same mechanism.

In referring to the two types of persistence, we have avoided the terms "Type I" and "Type II." While unambiguous in their original definitions, the terms have become ambiguous in more recent usage. The terms were first introduced by Hawkins and Shulman (1979) to distinguish between two different measures (rate of decay and total duration) of the same process, and not between two different processes. This was a useful distinction that highlighted different aspects of the decaying sensory residual. Later, the terms were redefined (by Long, 1979) as denoting qualitatively different kinds of persistence produced by separate mechanisms: off-responses and prolonged photoreceptor activity. In fact, it would be more accurate to say that the two terms were employed to denote not two, but three separate processes, in that "Type II persistence" was said to consist of two separate icons: one produced by the rods, the other by the cones. The resulting ambiguity has been further discussed by one of us (Di Lollo, 1983), in whose opinion further confusion would be avoided if the use of the two terms in connection with visible persistence were eschewed.

Besides, use of the two terms may be construed as suggesting that there exist only two types of visual aftereffects broadly classifiable as "persistence." To the contrary, we believe that there are many such aftereffects, visible and nonvisible, produced in the course of visual information processing. This follows from our conviction, outlined above, that visible persistence is the outcome of sensory coding activity within the visual system.

In a general sense, as the processing of the initial stimulus proceeds from energy transduction at the retina to the emergence of features and objects at more central levels, each stage would produce its own persistence, visual though not necessarily visible, always coded in terms of the processing activity prevalent at that stage. This clearly implies that persistence cannot be classified within a single category (e.g., iconic memory) or even within two categories (as illustrated in the present work). Instead, we suggest (as did Di Lollo, 1980) that there are as many expressions of sensory persistence as there are discernible phases of information processing.

\section{REFERENCES}

Adelson, E. H., Jonides, J. (1980). The psychophysics of iconic storage. Journal of Experimental Psychology: Human Perception \& Performance, 6, 486-493.

AllPort, D. A. (1968). Phenomenal simultaneity and the perceptual moment hypothesis. British Journal of Psychology, 59, 395-406.

Banks, W. P., \& Barbar, G. (1977). Color information in iconic memory. Psychological Review, 84, 536-546.

BREITMEYER, B. G. (1984). Visual masking: An integrative approach. New York: Oxford University Press.

Brindley, G. S. (1962). Two new properties of foveal after-images and a photochemical hypothesis to explain them. Journal of Physiology, 164, 168-179.

Brown, J. L. (1965). Afterimages. In C. H. Graham (Ed.), Vision and visual perception (pp. 479-503). New York: Wiley.

Coltheart, M. (1980). Iconic memory and visible persistence. Perception \& Psychophysics, 27, 183-228.

CraIK, K. J. W. (1940). Origin of visual afterimages. Nature, 145, 512.

DI LoLlo, V. (1980). Temporal integration in visual memory. Journal of Experimental Psychology: General, 109, 75-97.

Di LoLLo, V. (1983). On laterality of visual aftereffects: A rejoinder. Perception \& Psychophysics, 33, 599-603.

Di LoLLo, V., \& FinLEY, G. (1986). Equating the brightness of brief visual stimuli of unequal duration. Behavior Research Methods, Instruments, \& Computers, 18, 582-586.

EFRON, R., LEE, D. N. (1971). The visual persistence of a moving stroboscopically illuminated object. American Journal of Psychology, 84, 365-375.

ERIKSEN, C. W., * Collins, J. F. (1967). Some temporal characteristics of visual pattern perception. Journal of Experimental Psychology, 74, 476-484.

FinLEY, G. (1985). A high-speed point plotter for vision research. Vision Research, 25, 1993-1997.

Hawkins, H. L., \& Shulman, G. L. (1979). Two definitions of persistence in visual perception. Perception \& Psychophysics, 25, 348-350.

HoGbeN, J. H., \& Di LoLLo, V. (1974). Perceptual integration and perceptual segregation of brief visual stimuli. Vision Research, 14, 1059-1069.

IRWIN, D. E., \& Yeomans, J. M. (1986). Persisting arguments about visual persistence: Reply to Long. Perception \& Psychophysics, 39 , 225-230.

LoNG, G. M. (1979). Comment on Hawkins and Shulman's Type I and Type II visual persistence. Perception \& Psychophysics, 26, 412-414.

LoNG, G. M. (1980). Iconic memory: A review and critique of the study of short-term visual storage. Psychological Bulletin, 88, 785-820.

LONG, G. M., \& SAKITT, B. (1980). The retinal basis of iconic memory: Eriksen and Collins revisited. American Journal of Psychology, 93, 195-206.

Meyer, G. E., Maguire, W. M. (1977). Spatial frequency and the mediation of short-term visual storage. Science, 198, 524-525.

NeIsser, U. (1967). Cognitive psychology. New York: AppletonCentury-Crofts.

SAKITT, B. (1975). Locus of short-term visual storage. Science, 190, 1318-1319.

SAKITt, B. (1976). Iconic memory. Psychological Review, 83, 257-276.

UTTAL, W. R. (1981). A taxonomy of visual processes. Hillsdale, NJ: Erlbaum.

(Manuscript received December 15, 1987; revision accepted for publication February 10, 1988.) 